\title{
Redes de energia elétrica inteligentes (Smart Grids)
}

\author{
Intelligent electric power networks (Smart Grids) \\ Redes inteligentes de energía eléctrica (Redes Inteligentes)
}

Recebido: 16/07/2021 | Revisado: 19/07/2021 | Aceito: 19/07/2021 | Publicado: 19/07/2021

Verônica Dias Moreira Gallotti

ORCID: https://orcid.org/0000-0002-5082-1283

Universidade Federal do Rio de Janeiro, Brasil

E-mail: veronicagallotti@poli.ufrj.br

\begin{abstract}
Resumo
No país e no mundo, as Rede Elétricas Inteligentes situam-se no momento atual em uma etapa de crescimento embrionário, parecido ao da internet no início dos anos 1980, tendo ainda muitas viabilidades para os consumidores, empresas, concessionárias de energia, governos, fornecedores de tecnologia e desenvolvimento de novas capacitações na chamada "economia sustentável". Há ainda algumas dúvidas e incertezas sobre a regulação para o estabelecimento de legislação adequada à comercialização no varejo de energia elétrica para permitir o uso adequado de precificação dinâmica e da energia da microgeração. O propósito deste artigo é discutir e expor sobre Redes Elétricas Inteligentes ou Smart Grid, no inglês, seu conceito, propósito, componentes. Também sobre a regulamentação, o que deve ser mudado para se adaptar a essas tecnologias novas e suas medições modernas. O método de pesquisa utilizado foi o descritivo, onde foram usadas as técnicas de coleta de dados através de buscas em bibliografias reconhecidas e artigos, para assim, elucidar e exemplificar melhor sobre as Rede Elétricas Inteligentes. Por fim, conclui-se como deve ser feita a massificação dessa tecnologia no Brasil para ser ágil e eficiente.
\end{abstract}

Palavras-chave: Redes Elétricas Inteligentes; Smart Grid; Medição; Smart City; Transformação Energética.

\begin{abstract}
In the country and in the world, the Intelligent Electricity Grid is currently in a stage of embryonic growth, similar to the internet in the early 1980s, still having many possibilities for consumers, companies, energy concessionaires, governments, suppliers technology and the development of new capabilities in the so-called "sustainable economy". There are still some doubts and uncertainties about the regulation for the establishment of adequate legislation for the commercialization of electric energy in order to allow the adequate use of dynamic pricing and of microgeneration energy. The purpose of this article is to discuss and expose about Intelligent Electric Networks or Smart Grid, in English, its concept, purpose, components. Also about regulation, what must be changed to adapt to these new technologies and their modern measurements. The research method used was descriptive, where data collection techniques were used through searches in recognized bibliographies and articles, in order to elucidate and better illustrate the Intelligent Electric Network. Finally, it is concluded how the massification of this technology in Brazil should be done in order to be agile and efficient.
\end{abstract}

Keywords: Intelligent Electric Networks; Smart Grid; Measurement; Smart City; Energy Transformation.

\section{Resumen}

En el país y en el mundo, la Red Eléctrica Inteligente se encuentra actualmente en una etapa de crecimiento embrionario, similar a internet a principios de la década de 1980, aún teniendo muchas posibilidades para consumidores, empresas, concesionarias de energía, gobiernos, proveedores de tecnología y el desarrollo de nuevas capacidades en la llamada "economía sostenible". Aún existen algunas dudas e incertidumbres sobre la regulación para el establecimiento de una legislación adecuada para la comercialización de energía eléctrica que permita el uso adecuado de la tarificación dinámica y de la energía de microgeneración. El propósito de este artículo es discutir y exponer sobre Redes Eléctricas Inteligentes o Smart Grid, en inglés, su concepto, propósito, componentes. También sobre la regulación, qué hay que cambiar para adaptarse a estas nuevas tecnologías y sus modernas medidas. El método de investigación utilizado fue descriptivo, donde se utilizaron técnicas de recolección de datos mediante búsquedas en bibliografías y artículos reconocidos, con el fin de dilucidar e ilustrar mejor la Red Eléctrica Inteligente. Finalmente, concluye cómo debe hacerse la masificación de esta tecnología en Brasil para que sea ágil y eficiente.

Palabras clave: Redes eléctricas inteligentes; Smart Grid; Medición; Ciudad inteligente; Transformación energética. 


\section{Introdução}

As Redes Elétricas Inteligentes (REIs) são percebidas como a rede elétrica que utiliza tecnologia digital avançada para observar e gerenciar o transporte de eletricidade em tempo real com fluxo de energia e de informações bidirecionais entre o sistema de fornecimento de energia e o cliente final. A inserção das REIs proporciona uma variação de serviços novos.

A introdução das REIs no Brasil possuiria como fundamentais motivações: a procura das eficiências comercial e energética, a segurança operacional e sistêmica, o aumento da confiabilidade do sistema elétrico e a sustentabilidade econômica e ambiental.

A eficiência comercial e energética seria alcançada através da diminuição de perdas técnicas e comerciais, aperfeiçoamento na característica da energia ofertada ao consumidor e comando do horário da consumação de energia pelo consumidor.

A segurança operacional e sistêmica seria obtida através do controle de acesso dos usuários de rede, da diminuição de energia não distribuída, das perdas por fraudes, tolerância a ataques físicos e cibernéticos, desta forma a diminuir a interrupção no fornecimento e os custos relativos ao restabelecimento do sistema. Ademais, segurança para as pessoas que mexem com a rede (consumidores e as equipes de manutenção).

A confiabilidade se dá pela prevenção dos problemas relacionados aos equipamentos (técnicas de manutenção baseadas na condição) e competência de suportar distúrbios (minimização da interrupção no fornecimento).

Finalmente, a sustentabilidade econômica e ambiental seria resultado da modificação dos negócios e das viabilidades para novos serviços de custo agregado concedidos pelas concessionárias, assim como a atual regulamentação do net metering e o uso de veículos elétricos e híbridos conectados às redes.

Um incentivo comum a todas as empresas distribuidoras para a inserção de smart grids é o aparecimento de carros com tração elétrica. Com o crescimento do valor dos combustíveis fósseis, levando em conta as consequências no meio ambiente decorrente do uso de fontes não renováveis de energia, e considerando os custos decrescentes das baterias, o aumento do uso de carros com tração elétrica, principalmente nos grandes centros urbanos, é uma tendência apontada como irreversível. E, principalmente, calcula-se que o uso deste tipo de veículo pelas pessoas deve-se popularizar rapidamente. Assim, as distribuidoras de energia elétrica estão preocupadas com o abrupto acréscimo da carga nas grandes cidades devido ao crescimento do uso de carros de tração elétrica, ou seja, o aumento do consumo de energia elétrica decorrente da demanda associada à recarga das baterias dos carros de tração elétrica.

A forma técnica e economicamente viável para absorção desta carga complementar pelos sistemas existentes é o gerenciamento da demanda adicional associada ao carregamento das baterias dos automóveis a partir da definição de tarifas diferenciadas que proporcionem tal carregamento quando o sistema de fornecimento de energia elétrica esteja sendo menos exigido pelas cargas tradicionais.

Adicionalmente, percebeu-se que a energia armazenada nas baterias dos automóveis elétricos estacionados pode ser reinjetada no sistema elétrico em casos de emergência ou nos horários de pico de demanda. Essas ações requisitam a aplicação da tecnologia que descreve as smart grids, ou seja, tarifas variáveis, conhecimento sobre tarifas e desempenho da rede disponíveis em tempo real para os usuários, medidores de energia inteligentes, utilização de fontes alternativas de energia, sistemas de armazenagem de energia, e consumidores que podem aumentar seu consumo ou mesmo injetar energia na rede em função das informações disponibilizadas na rede. Todas essas ações ainda estão sendo implementadas, tanto no Brasil quanto em outros países do mundo, uns já estão mais avançados do que outros. No Brasil, já existem algumas cidades “smarts”, porém as regulamentações precisam ser mais adaptadas ainda sobre o assunto.

Em síntese, e para fins deste artigo, o propósito é mostrar os motivos que estão levando a modernização das redes elétricas, a importância das Redes Elétricas Inteligentes, discutir e expor seu conceito, o que tem feito até agora, como a smart 
city no Brasil em São Paulo, na Vila Olímpia e como são feitas as medições nessas novas redes elétricas. Tendo a finalidade, então, de apresentar essa nova mudança para os interessados.

\section{Metodologia}

Para o desenvolvimento e obtenção dos resultados apresentados neste trabalho, utiliza-se a metodologia exploratória de coleta de informações e dados (MOTA-ROTH e HENDGES, 2010). São feitos levantamentos em fontes secundárias objetivando reunir informações, mas sem intenção de testar hipóteses, e baseando-se em obras de importantes autores e pesquisas em órgãos da área presentes no País. Enfatiza-se a busca por conceitos, pensamentos e abordagens nas obras dos autores pesquisados, em busca de definições que delimitem os conceitos de REIs, resposta da demanda e transformação energética. Além disso, são sugeridas possíveis maneiras de como proceder na inserção das REIs no Brasil através de um plano nacional estruturado, abrangendo agências normatizadoras e reguladoras

\section{Transformação Energética}

A Transformação Energética é uma das prioridades mundiais, pois as transformações são constantes e imprescindíveis para a evolução e desenvolvimento da sociedade. A produção, o consumo de energia renovável, automação, gerenciamento do consumo de indústrias, mobilidade elétrica, investimentos em digitalização de redes elétricas de média e baixa tensão, comércios e residências, ademais de novas tecnologias de armazenamento e geração de energia descentralizadas são alguns exemplos de tendências que orientam o futuro do setor energético mundial. A transformação energética foi denominada de 3Ds, uma vez que tem como base a Descentralização, Digitalização e Descarbonização.

Na Descentralização, o consumidor também é gerador de energia, com o poder de tomar decisão de consumo em tempo real. Já na Digitalização, permite o maior controle de dados em tempo real tanto para a distribuidora e comercializadoras, quanto para os consumidores. As Smart Grids são as digitalizações, que utilizam tecnologia da informação para fazer o sistema ser mais eficiente, seus componentes são: medidores inteligentes, sensores inteligentes, sincrofazores, automação, Machine Learning, Big Data and Analytics e Blockchain. A Descarbonização é a substituição de termelétricas por fontes renováveis, reduzindo o uso de combustíveis fósseis.

O sistema elétrico do futuro é caracterizado por:

- geração variável e distribuída;

- estabilidade reduzida devido às novas fontes;

- $\quad$ sistema de controle distribuído, flexível e resiliente;

- dinâmica muito mais rápida;

- carga flexível e transacional;

- modelo de negócios baseado em serviços;

- resposta a crescente expectativa dos clientes em relação à qualidade do fornecimento de energia, assim como, aos anseios do regulador e das demais autoridades;

- a tendência de competição no mercado de energia elétrica direta ou indiretamente;

- e a necessidade da diminuição de custos operacionais, como, exemplificando, aqueles relativos a perdas e inadimplência.

Esse novo cenário com a geração distribuída, eólica solar, gerenciamento da demanda, veículos elétricos e mudanças climáticas causam maior variabilidade, menor previsibilidade e menor despachabilidade. 
De forma resumida, os principais objetivos e necessidades para a modernização das redes elétricas são listadas como (Arnold, 2011):

- Possibilitar de forma confiável e estável a expansão da capacidade de fornecimento de eletricidade, mediante a crescente demanda;

- Melhorar a relação custo-benefício nos processos de produção e de fornecimento de energia elétrica;

- Prover informações ao consumidor sobre o consumo individual de energia elétrica, bem como prover ferramentas para auxiliar no controle e na tomada de decisão acerca do consumo;

- Auxiliar na redução da emissão de gases decorrentes da produção de energia elétrica com recursos fósseis

- Melhorar os níveis de confiabilidade dos serviços ofertados;

- Explorar novos nichos mercadológicos no setor, por meio do fornecimento de novos produtos e serviços;

- Operar de forma resiliente em situações de ataque ou de desastres naturais;

- Antecipar e responder a perturbações no sistema mediante reconfiguração automática;

- Preparar a rede elétrica para suportar a crescente demanda futura de veículos elétricos;

- Dotar a rede de dispositivos de armazenamento de energia para uso conjunto com fontes intermitentes

\section{Redes Elétricas Inteligentes}

Para essa modernização da rede elétrica apresentada no capítulo anterior, as Redes Elétricas Inteligentes (REI) conhecido também como Smart Grid (SG) é a nova forma de atender a esses requisitos. É uma rede elétrica com uma grande integração de tecnologia de informação, telecomunicações, sensoriamento/medição e automação, de forma a expandir consideravelmente sua competência de atender cenários com fontes intermitentes e distribuídas de energia, altos requisitos de confiabilidade, baixo impacto no meio ambiente e compatível com novos mercados de energia. Os seus componentes são sensores e atuadores (medidores inteligentes, medição fasorial sincronizada, inteligent eletronic devices e chaves automáticas), recursos energéticos distribuídos (microgeração, minigeração, geração distribuída, armazenamento de energia), automação e controle (religadores e reguladores de Tensão Inteligentes), eletrificação de transportes (veículos elétricos), aplicações dos consumidores (eletrodomésticos inteligentes, sistemas domésticos de gerenciamento de energia).

Como o conceito de REI é muito extenso, não tendo uma única definição preferida por diferentes governos, o propósito de seu progresso também varia. Por exemplo, nos EUA a finalidade fundamental é tratar o envelhecimento de suas redes, aprimorar a capacidade de serviço, promover empregos e fomentar a indústria e ampliar a interação com o usuário. $\mathrm{O}$ ponto central na Europa é o de proporcionar o emprego de energias renováveis e diminuir a subordinação de combustíveis fósseis para uma maior segurança energética.

No caso do Brasil, o propósito pode ser visto como juntar tanto os motivos americanos quanto os europeus sob os pontos de vista das concessionárias, dos consumidores e do agente regulador, com ênfase para:

- diminuir as perdas técnicas e comerciais (fraudes);

- aprimorar a qualidade do serviço prestado pelas distribuidoras;

- reduzir os custos operacionais;

- melhorar o planejamento da expansão da rede;

- aperfeiçoar a gestão dos ativos;

- impulsionar a eficiência energética;

- desenvolver a inovação e a indústria tecnológica. 
Os impactos das redes elétricas inteligentes em cada parte da energia elétrica são diversos: na geração, seu impacto é pequeno, já na área da transmissão é moderado, em relação à distribuição é grande (sendo o segmento no qual as tecnologias de REI têm tido maior aplicação) e no consumo é revolucionário, incluindo a geração distribuída (micro e mini).

As redes elétricas inteligentes podem ajudar:

- nas fontes intermitentes de energia e armazenamento: eólica, solar, ondas, armazenamento, veículos elétricos;

- nas gerações distribuídas conectada na rede de distribuição: microgeração e minigeração;

- na melhoria da confiabilidade: automação da distribuição e microrredes;

- no gerenciamento pelo lado da demanda: resposta da demanda, reduzindo a ponta, e tarifação dinâmica;

- redução de perdas: perdas técnicas, perdas não-técnicas (furto e fraude); além de outros custos como grandes áreas de concessão, alimentadores radiais longos, áreas de difícil acesso e aplicações (faturamento, localização de faltas, self healing, monitoração de ativos).

\section{Resposta da Demanda}

Conforme a EPE, a resposta da demanda é um recurso que possibilita aos consumidores maior comando da utilização da energia elétrica, atendendo às mudanças no preço e aos controles do operador do sistema através de pagamentos. Com a frequente presença dos consumidores, o sistema elétrico consegue obter uma sucessão de benefícios, podendo-se citar:

- Maior flexibilidade e confiabilidade;

- Menos lançamento de gases de efeito estufa;

- Atenuação dos gastos de geração, visto que com a redução da demanda no horário de ponta, térmicas mais dispendiosas não precisarão ser tão demandadas, ocasionando privilégios econômicos mesmo para os compradores que não estão contribuindo para programas de resposta da demanda;

A smart grid, em união com a disperção dos smart meters (medidores inteligentes que possibilitam a comunicação bidirecional, proporcionando despacho de dados em duas vias, no meio dos pontos de consumo e a concessionária), modificará a resposta da demanda, evoluindo a habilidade de geradores e consumidores de eletricidade de se transmitirem e tomarem medidas sobre quando e como gerar e consumir energia. Essa tecnologia proporciona que os clientes mudem de um modelo de resposta da demanda sustentado em mudanças, assim o consumidor enxerga o sinal para poder modificar sua demanda a todo momento, transformando-se em um agente ativo do sistema.

Existem dois tipos de programas de resposta da demanda que podem ser implementados, são eles:

Resposta da demanda baseada em preços: relaciona-se à transformação na representação de uso da energia por causa das mudanças no valor durante as horas do dia. Assim, o consumidor muda o gasto da energia para instantes em que o preço está menor e minimiza o uso nos momentos em que o preço está mais elevado. O período de horas, estipulado pelas concessionárias, no qual o custo da energia é maior coincide ao instante em que a carga total da concessionária alcança valores mais altos e o atendimento está mais caro.

Resposta da demanda baseada em incentivos: Este tipo de resposta disponibiliza aos clientes benefícios financeiros para atenuamento do consumo em situações críticas do sistema, quando a proposta se encontra insuficiente ou quando há caimento da confiabilidade. São produtos despacháveis, isso quer dizer, são acionados por meio de uma ordem de despacho do operador. Esta prática é oferecida à parte do modelo de tarifação e pode acontecer ao mesmo tempo em que o consumidor esteja sob tarifação dinâmica. Os consumidores diminuem seu gasto em relação a uma linha base determinada previamente e são punidos se não conseguirem atingir o uso energético anteriormente definido. 


\section{Aspectos Regulatórios e Leis}

Com essas novas tecnologias, novos arranjos comerciais surgindo, uma nova regulamentação será necessária para reorganizar e redistribuir os custos e os benefícios dessas transformações entre consumidores e agentes envolvidos.

É essencial, para isso, desenvolver, ou até mesmo mudar, a regulação atual para diminuir as barreiras percebidas pelas distribuidoras no que se refere à perda de receita com a diminuição da utilização de eletricidade pelos seus consumidores finais. Elas têm que ser incentivadas a perceber a eficiência energética e a geração distribuída como oportunidade de negócios e de novas receitas. Deve ser apontada, ainda, a participação de novas empresas que podem ou não concorrer com as distribuidoras. Os consumidores poderiam, então, visualizar as oportunidades de reduzir seus gastos com eletricidade e de ter um papel mais ativo junto à distribuidora.

A busca do equilíbrio dessas relações entre os consumidores, as concessionárias e o governo é a função da regulação. As modificações na regulação setorial serão imprescindíveis, tendo as seguintes mudanças:

- a redefinição das tarifas;

- Restauração dos investimentos gerados anteriormente e dos investimentos essenciais para a inserção de smart grids;

- a criação de normas referentes a armazenagem de energia, a novos eletrodomésticos, processos industriais, geração distribuída; e

- a determinação de protocolos e métodos para assegurar a proteção das informações que transitarão na rede de fornecimento de energia elétrica, sendo indescritível para a viabilização das smart grids.

A regulação deve acompanhar as transformações de modo a manter o equilíbrio econômico e financeiro de todos os envolvidos e, inclusive, refletir nas tarifas a repartição dos custos da manutenção do sistema interconectado e sinalizar oportunidades de novos investimentos onde forem necessários.

O estudo feito pela Abradee (2011) diz que para que as vantagens se tornam percebidas, seriam essenciais ajustes no arcabouço regulatório atual para analisar as vantagens concedidas a: microgeração distribuída, desenvolvimento na propriedade do serviço, perda da energia não distribuída, atenuaação dos investimentos de expansão da geração e transmissão e remuneração e depreciação dos investimentos realizados no período.

As mudanças que devem ocorrer são (Abradee, 2011):

- Subsídio do investimento em REIs - conforme o estudo, o investimento nas Rede Elétricas Inteligentes não tem recompensa para as distribuidoras nas circunstâncias regulatórias vigentes.

- Mudança na regulação sobre os lucros auferidos em recentes negócios por meio das REIs - a regulação presente só concede que as concessionárias obtenham 10\% dos lucros na prestação de serviços diferentes da comercialização de energia elétrica.

Em termos nacionais, normas e regulamentações ainda deparam-se em fase de desenvolvimento e adaptação, uma vez que inicialmente podemos destacar o trabalho do Ministério de Minas e Energia - MME, que com a edição da Portaria n 440 , de 15 de abril de 2010, formou uma equipe para estudar e reconhecer comportamentos fundamentais para custear a formação de políticas públicas para a inclusão de um Programa Brasileiro de REI.

A regulamentação necessária à implantação dessas tecnologias e de novos serviços e tarifas é de responsabilidade da ANEEL, atualmente, ao passo que as normas contendo as especificações e métodos de ensaios para os novos medidores eletrônicos inteligentes exigem a atuação da ABNT, sendo que a certificação é de compromisso do INMETRO. Logo, existe uma série de leis, órgãos, regulamentos e normas vinculadas a esse novo conceito de redes de energia elétrica, evidenciando:

- Leis e decretos federais: relativos ao fornecimento de energia elétrica e ao modelo tarifário vigente no país; 
- Regulamentos da ANEEL: tratam das condições gerais de fornecimento de energia elétrica e uma série de resoluções voltadas à medição de energia elétrica;

- Regulamentos do INMETRO: tratam dos requisitos técnicos e metrológicos que os sistemas de medição centralizada devem satisfazer para uso de medição de energia elétrica em unidades consumidoras estabelecendo obrigações para distribuidoras e fabricantes;

- Normas da ABNT: voltadas aos medidores eletrônicos e ou inteligentes, que tratam de especificações, métodos de ensaio, procedimentos gerais e padronização.

Conforme as REI foram crescendo no Brasil foram sendo criados grupos de trabalho e estudo, são eles:

- o grupo de trabalho do MME (Ministério de Minas e Energia), formado por uma comissão de distribuidoras, reguladores do setor elétrico, reguladores do setor de telecomunicações, associações, órgãos financiadores, tecnologia da informação e setor acadêmico. Tem como objetivo apresentar as possibilidades e desafios a serem vencidos para implementação de novas tecnologias na rede elétrica.

- grupo de estudos da CGEE/MCTI que tem o estudo encomendado pelo MCTI com objetivo de congregar uma visão panorâmica sobre as transformações nas redes de energia elétrica e subsidiar a inserção do tema na política de C\&T em energia.

- diálogo setorial Brasil-União Europeia que tem como propósito a identificação de projetos relevantes no Brasil e na Europa na área de Redes Elétricas Inteligentes (REI), informações sobre os fabricantes e fornecedores dos equipamentos que compõem a infraestrutura de REI no Brasil e na Europa, identificação de instituições públicas e privadas no Brasil e na Europa, que atuaram, estão atuando ou pretendem atuar, em projetos relacionados ao tema de REI, análise das políticas industriais e de ciência e tecnologia no Brasil e na Europa.

Nos últimos anos, além do MME, embora até recentemente não haver uma política nacional para as redes inteligentes de energia elétrica, a ANEEL, igualmente apresenta variadas funções relacionadas à implantação de REIs no país, como por exemplo:

- atitudes orientadas para a inserção de medidores eletrônicos inteligentes em residências;

- criação das condições gerais para o acesso de microgeração e minigeração distribuída aos sistemas de distribuição de energia elétrica juntamente com sistema de compensação de energia elétrica;

- e alterações no arranjo tarifário aspirando a mudança horária e sazonal da tarifa

\section{Smart Cities e seus Programas Incentivadores}

A possibilidade da fixação dos conceitos "smart" - como REIs e cidades inteligentes (smart cities) - está estreitamente relacionada aos progressos tecnológicos da eletrônica, com incremento dos aparelhos de sensoriamento, geração de energia distribuída e armazenamento de dados e da evolução nos sistemas de controle. Esse fato proporcionará a inserção de cidades inteligentes, além de ambientes onde todos os objetos podem ser unicamente identificados, reconhecidos, localizados e endereçados, isto vem sendo denominado de Internet of Things (IoT) - ou Internet das Coisas

Para subsidiar o estabelecimento das redes inteligentes e seus projetos, a princípio, até que seja definida uma política nacional e formas para financiar ou amortizar tais investimentos, as empresas de energia elétrica estão utilizando recursos dos programas obrigatórios de P\&D e PEE (Pesquisa e Desenvolvimento e de Eficiência Energética), onde as mesmas são obrigadas a aplicar cerca de $1 \%$ da receita operacional líquida anual nestes dois Programas. Os projetos são propostos e desenvolvidos pelas próprias empresas a seu exclusivo critério, mas dentro de regras estabelecidas pela Agência, que objetivam garantir uma efetividade mínima destes investimentos. Segundo Boccuzzi: 
Estes programas, até agora, se constituíam na fonte de recursos disponível para o desenvolvimento das redes inteligentes e vem sendo utilizados para a implantação de mais de 10 projetos de cidades inteligentes em igual número de empresas, de um total de 63 distribuidoras existentes no país. Todas as empresas, sem exceção e com diferentes focos, aderentes às suas realidades de mercado e de sistemas, estão investindo na busca de tecnologias de redes inteligentes que possam oferecer retorno econômico e eficiência operacional.

Um dos projetos que utiliza esses programas para sua implementação é o projeto Urban Futurability, que teve lançamento, em São Paulo, no dia 9 de outubro de 2019 e tem como objetivo a transformação digital da rede elétrica, prometendo tornar a Vila Olímpia, Zona Sul da capital paulista, em um laboratório de mais de 40 iniciativas de digitalização e inteligência artificial, inéditas no país e até na América do Sul. A ideia é ter outras cidades de São Paulo também beneficiadas por essas tecnologias. São Paulo foi escolhida por ser uma das maiores áreas urbanas da América Latina de acordo com Francesco Starace, CEO da Enel.

A iniciativa contempla a criação de uma réplica digital tridimensional da rede elétrica da Vila Olímpia e a instalação de aproximadamente 4.900 sensores, que coletarão dados sobre as condições da rede. Os dados, disponibilizados em tempo real, serão capazes de gerar novas oportunidades, criar valor à sociedade, ao município, utilities e outras empresas, criando um pool de iniciativas capaz de tornar sustentável uma megacidade como São Paulo.

Os dados impulsionam também novos serviços e soluções aos cidadãos, universidades, municípios e startups, respondendo às necessidades de infraestrutura local, como, por exemplo, mobilidade urbana, segurança, redução de resíduos e plantação urbana, gerando novas oportunidades de negócios.

O projeto será implementado em parceria com as autoridades governamentais locais e a Agência Nacional de Energia Elétrica (ANEEL). Serão investidos cerca de R\$ 125 milhões nos próximos três anos, com recursos do programa de Pesquisa e Desenvolvimento (P\&D) da Aneel. O objetivo do Programa de P\&D é alocar adequadamente recursos humanos e financeiros em projetos que demonstrem a originalidade, aplicabilidade, relevância e a viabilidade econômica de produtos e serviços, nos processos e usos finais de energia. Busca-se promover a cultura da inovação, estimulando a pesquisa e desenvolvimento no setor elétrico brasileiro, criando novos equipamentos e aprimorando a prestação de serviços que contribuam para a segurança do fornecimento de energia elétrica, a modicidade tarifária, a diminuição do impacto ambiental do setor e da dependência tecnológica do país.

O processo de escaneamento captura imagens em alta resolução e termográfica dos ativos elétricos nos arredores, essas imagens se transformam em modelo 3D fiel da rede. O modelo 3D digital permite a medição precisa e remota das rede elétricas áreas, subterrâneas e do ambiente em que estão inseridas. Ele também possibilita a identificação de interferências. Com inteligência artificial pode-se reconhecer automaticamente os ativos presentes no modelo. De acordo com a ANEEL ativos são máquinas, materiais e equipamentos destinados à prestação de serviços de eletricidade. Essa inteligência permite uma visão da rede em tempo real, possibilitando inclusive identificação antecipada de anomalias. Essas anomalias são enviadas para o centro de controle da Enel Distribuição São Paulo. No centro de distribuição são analisados os riscos de forma preventiva e assertiva, toda essa tecnologia contribui para a criação do Network Digital Twin que também abre portas para outras possibilidades. Os dados digitalizados pela Enel podem ser compartilhados com outros Stakeholders, como municípios, empresas e clientes para criação de novos serviços. Com isso, o urban futurability através do Network Digital Twin colabora para a formação de uma megacidade sustentável.

Os benefícios são muitos, com todos esses dados que vão ser levantados disponíveis, vai ficar mais fácil antecipar problemas. Além disso, sistemas inteligentes identificarão também outros ativos que interfiram na operação, como árvores próximas aos cabos, o projeto possibilitará também o engajamento de outros entes como o poder público e as empresas de serviços, uma vez que o mapa digital da rede terá informações também de ativos de telecomunicações, de iluminação pública, 
semáforos, além de reduzir quedas de energia e otimizar a atuação durante ocorrências. Outro benefício, é que o projeto busca materiais sustentáveis, as câmaras dos transformadores subterrâneos, que hoje são de concreto, passarão a ser feitas, principalmente, de plástico reciclado. Também está em desenvolvimento a troca dos transformadores com óleo mineral por outros com óleo vegetal ou sem óleo nenhum.

A partir do monitoramento do Centro de Controle, será possível enviar equipes para efetuar reparos, reduzindo de forma significativa o tempo de atendimento e o número de clientes interrompidos. Para isso, foi necessário introduzir no projeto diversos tipos de sensores e estruturas, como fibra ótica e comunicações desenvolvidas para IoT. Isso servirá para alimentar um grande sistema de inteligência artificial que vai monitorar como a rede de distribuição convive com os demais ativos utilizados pelo município e outras empresas, com o objetivo de propor o melhor plano de manutenção preventiva.

Outro benefício, é que todo esse monitoramento e otimização de serviço será feito de forma segura e rápida. A digitalização dos processos, por meio da realidade aumentada, somada à disponibilidade de dados, aumentarão a segurança e eficiência das operações de campo, acelerando o diagnóstico do problema e oferecendo mais rapidamente uma solução assertiva. A imersão digital em simulações de atividades de treinamento em um ambiente completamente virtual reduzirá riscos e disponibilizará feedbacks em tempo real, possibilitando o aperfeiçoamento e aprendizado cognitivo.

\section{Medição das Smart Grids}

Para muitos, a Infraestrutura Avançada de Medição é o elemento fundamental para implantação do conceito de Smart Grid. Inclusive, a maioria dos projetos de Smart Grid inicia-se pela implantação de uma IAM. Uma IAM é uma arquitetura para comunicação automatizada, de duas vias entre medidores inteligentes, com endereços IP, e uma empresa de serviços públicos. A finalidade é dispor empresas de serviços públicos com dados em tempo real sobre o consumo de energia e conceder aos clientes o poder de fazer escolhas racionais sobre o uso de energia com base no preço no instante do aproveitamento.

O sistema convencional de medição de energia é caracterizado pelas seguintes propriedades: coleta mensal, medidor eletromecânico ou eletrônico, leitura manual e processamento manual ou automático e, também, por banco de dados.

Com o passar dos anos, a medição passou a ser mais automática, sendo chamada de Automated Meter Reading (AMR) no inglês, sua particularidade é a coleta em quase tempo-real, comunicação mono-direcional, medidor eletrônico, leitura automática, processamento automático e banco de dados.

Em seguida, teve mais avanços com as tecnologias e a medição passa a ser chamada de Infraestrutura de Medição Automatizada (da sigla em inglês Advanced Metering Infrastructure - AMI) através dessa implantação terá as seguintes mudanças no sistema elétrico (Oklahoma Gas and Electric Company, 2013) e (Faruqui, 2009):

- Comunicação em duas vias - Com a instalação de smart meters será capaz de obter o fluxo de dados em duas vias. Isso quer dizer que, nos dias atuais, o fluxo de informação flui dos consumidores para as concessionárias e o fluxo de energia flui das concessionárias para os consumidores. Com a implantação desta tecnologia os tipos de fluxo (informação e energia) poderão fluir nos dois sentidos, sendo uma comunicação bi-direcional;

- Diminuição dos custos de medição - Por não ser preciso uma equipe para a execução de medição em residências com os sistemas automatizados;

- Tempo de conexão/reconexão - A concessionária saberá em tempo real quais unidades consumidoras estão sofrendo dificuldade, não necessitando que os usuários façam contato com a empresa para comunicar a ocorrência de problemas, reduzindo assim o tempo de religamento da unidade;

- Postergar/evitar construção de novas usinas - Com a redução da demanda de ponta e energia por meio da resposta da demanda, a construção de usinas poderá ser postergada ou até mesmo evitada; 
- Diminuição dos custos de geração - com a diminuição da demanda de ponta o custo de geração neste horário ficará menor. Em horários de grande demanda são despachadas também as usinas com custo de geração mais alto, geralmente termelétricas, fazendo com que o custo de geração eleve. Reduzindo o consumo na ponta, menos termelétricas caras serão despachadas, fazendo com que o custo de geração do sistema caia;

- Redução de emissão de CO2 - Para satisfazer a demanda do sistema são despachadas as usinas mais caras, que em sua maioria possuem uma maior relação $\mathrm{CO} 2 / \mathrm{kWh}$. Com a redução da demanda no horário de ponta, a emissão global de $\mathrm{CO} 2$ do sistema elétrico cairá.

O sistema de monitoração e controle de segurança engloba atividades como monitoração de segurança, seleção e análise de contingências, controle preventivo, entre outras (VANTI, 2000; SOUZA, 2002). Hoje em dia, este sistema é implementado com base em dados provenientes de um conjunto de telemedidas redundantes, digitais e analógicas, que compõe o sistema SCADA ("Supervisory Control and Data Acquisition"). Estes dados, obtidos em intervalos regulares de tempo, são recebidos e processados através de ferramentas computacionais chamadas Configurador de Rede e Estimador de Estados, para que se conheça a configuração em tempo real do sistema e o perfil das tensões complexas nas barras. Contudo, o processamento destas medidas não é instantâneo, fazendo com que o processo não seja apto a proporcionar o estado real do sistema, e sim uma estimativa fundamentada na redundância das medidas do estado do sistema em um instante de tempo perto, entretanto antes do tempo real.

Dentre as novas tecnologias que vêm sendo propostas, a medição fasorial sincronizada tem recebido grande atenção de grupos de pesquisas e de empresas do setor elétrico, em âmbito mundial. Este novo sistema de medição destaca-se pelo uso das unidades de medição fasorial, mais conhecidas como PMUs ("Phasor Measurement Units"). Por utilizarem uma fonte eficaz de sincronização, fornecida pelo sistema GPS (“Global Positioning System”), as PMUs viabilizam a realização da medição de grandezas fasoriais em instalações geograficamente distantes, a uma taxa de até 60 medidas por segundo e com precisão angular adequada aos requisitos das aplicações, possuem taxa de atualização (varredura) muito maior que o sistema SCADA e permite a monitoração e o controle dinâmico do sistema elétrico. Tais características vêm de encontro às necessidades tecnológicas atuais e potencializam o desenvolvimento de novos paradigmas para a supervisão e controle do sistema em tempo real.

\section{Conclusão}

Este artigo apresenta as novidades da transição energética que vêm acontecendo com as Redes Elétricas Inteligentes, mostrando os principais objetivos e necessidades para a modernização das redes elétricas. Os componentes das REIs, seu conceito, apresentar a resposta da demanda com essas redes modernas, o que os estudos e autores dizem sobre o que deve ser mudado na regulação. Apresenta, também, projetos inovadores como as smarts cities, ilustrado aqui no artigo a Urban Futurability que está sendo desenvolvido no Brasil. Além de como são feitas as medições nas smarts grids, tudo isso com o propósito de expor e orientar a quem se interesse pelo assunto.

Destaca-se que há bastantes etapas a vencer para a ficxação completa do conceito de smart grid, que vai além da simples inserção de medidores inteligentes e do domínio dos projetos-piloto em andamento atualmente. E o Brasil, por ser um país grande geograficamente, em clima, sócio e culturalmente, revela barreiras distintas que, se por um lado retrata um obstáculo a ser superado, por outro compõe-se uma chance para inovações locais. Principalmente em domínios como software, telecomunicações, segurança, automação de rede e dispositivos avançados - microgeração, automação residencial, iluminação eficiente, veículos elétricos, armazenamento etc. - Existe um acerto grande de novidades que deverão ser expandidas conforme o andamento do recebimento e conhecimento dos consumidores ligados às REIs. 
Para que aconteça de fato a massificação das tecnologias brasileiras, é de extrema importância a inserção de um plano nacional estruturado para as REIs, abrangendo, ao menos, agências reguladoras e normatizadoras, por exemplo: Anatel, Aneel e Inmetro, órgãos financiadores, representantes da indústria, os ministérios (Ministério das Comunicações, MME, MDIC e MCTI) e respectivas agências a estas associadas.

Como sugestão para trabalhos futuros pode ser investigado detalhadamente como os países estrangeiros têm feito para incluir as Redes Elétricas Inteligentes no dia a dia, quais as leis feitas e os impactos na sociedade. Além de poder dar sugestão de quais leis foram bem-sucedidas e poderiam ser implementas no Brasil.

\section{Referências}

Agência Nacional de Energia Elétrica. (n.d.) Programa de pesquisa e desenvolvimento tecnológico do setor de energia elétrica. https://www.aneel.gov.br/programa-de-p-d

Arnold, G. (2011). Challenges and opportunities in smart grid: A position article. Proceedings of the IEEE, 99(6), $922-927$.

Associação Brasileira de Distribuidores de Energia Elétrica. (2011). Projeto estratégico de p\&d redes elétricas inteligentes: políticas públicas e regulação. FGV

Bajay, S., Jannuzzi, G. M., Heideier, R. B., Vilela, I. R., Paccola, J. A., \& Gomes, R. (2018). Geração distribuída e eficiência energética: Reflexões para o setor elétrico de hoje e do futuro. Campinas: IEI Brasil. 332p. https://iei-brasil.org/wp-content/uploads/2018/01/Gera\%C3\%A7\%C3\%A3odistribu\%C3\%ADda-e-efici\%C3\%AAncia-energ\%C3\%A9tica-Reflex\%C3\%B5es-para-o-setor-el\%C3\%A9trico-de-hoje-e-do-futuro.pdf

Bandeira, F. P. M. (2012). Redes de energia elétrica inteligentes (smart grids). Brasília: Consultoria Legislativa.

https://bd.camara.leg.br/bd/handle/bdcamara/9848

Boccuzzi, C. (2021) A modernização tecnológica e as recentes mudanças no setor elétrico brasileiro. www.smartgrid.com.br

CGEE. (2012). Redes elétricas inteligentes: contexto nacional. Brasília: Centro de Gestão e Estudos Estratégicos.

https://www.cgee.org.br/documents/10195/734063/Redes_Eletricas_Inteligentes_22mar13_9539.pdf/36f87ff1-43ed-4f33-9b53-5c869ace9023?version=1.1

Colak. I. (2016). Introduction to smart grid. 2016 International Smart Grid Workshop and Certificate Program (ISGWCP).

Ehrensperger, J. G. (2003). Sistema de medição fasorial: Estudo e análise do estado da arte. Florianópolis: Universidade Federal de Santa Catarina. http://www0.fee.unicamp.br/cursos/ET720/Cap-1-Apoio/UFSC-monografia.pdf

ENEL. (n.d.) Urban futurability: uma vila olimpia digital. https://www.eneldistribuicaosp.com.br/urban-futurability

EPE. (2019). Nota técnica - resposta da demanda: Conceitos, aspectos regulatórios e planejamento energético. https://www.epe.gov.br/sites-pt/publicacoesdados-abertos/publicacoes/PublicacoesArquivos/publicacao-389/NT_EPE_DEE-NT-022_2019-r0.pdf

Falcão, D. M. (2012, setembro) Redes elétricas inteligentes: Smart Grids. Inserção de novas fontes renováveis e redes inteligentes no planejamento energético nacional, Rio de Janeiro, RJ, Brasil.

IEA. (2020). Global EV outlook 2020. Paris: IEA. https://www.iea.org/reports/global-ev-outlook-2020

Li, S., Da Xu, L., \& Zhao, S. (2015). The internet of things: a survey. information systems frontiers, 17(2), $243-259$.

Motta-Roth, D., Hendges, G. R. (2010). Produção textual na universidade. Parábola.

Muller, G. de M. (2016). Impacto de novas tecnologias e smart grids na demanda de longo prazo do sistema elétrico brasileiro. (Tese de doutorado). Programa de engenharia elétrica, Universidade Federal do Rio de Janeiro, Rio de Janeiro, RJ. http://pee.ufrj.br/teses/textocompleto/2016032902.pdf.

Okai, E., Feng, X., \& Sant, P. (2018). Smart Cities Survey. 2018 IEEE 20th International Conference on High Performance Computing and Communications; IEEE 16th International Conference on Smart City; IEEE 4th International Conference on Data Science and Systems (HPCC/SmartCity/DSS).

Schlickmann, F. (n.d.). Redes inteligentes de energia elétrica: implantação, regulamentação e benefícios. São Paulo: Portal educação. https://siteantigo.portaleducacao.com.br/conteudo/artigos/administracao/redes-inteligentes-de-energia-eletrica-implantacao-regulamentacao-ebeneficios $/ 56232$

Souza, A. (2002). Segurança dinâmica de sistemas de energia elétrica sob o paradigma de serviços ancilares. (Proposta de Tese de Doutorado). Centro Tecnológico, Universidade Federal de Santa Catarina, Florianópolis.

Vanti, M. V. (2000). Uma formulação do fluxo de potência ótimo considerando restrições de estabilidade transitória. (Proposta de Tese de doutorado). Centro Tecnológico, Universidade Federal de Santa Catarina, Florianópolis. 